INRA Prod. Anim.,

2013, $26(1), 51-64$

\section{Prospective de la filière équine française à l'horizon 2030. Quatre scénarios contrastés pour éclairer la décision}

\author{
C. JEZ', B. COUDURIER ${ }^{2}$, M. CRESSENT ${ }^{3}, F . M E A^{4}$, P. PERRIER-CORNET
}

${ }^{1}$ INRA, UAR1241 DEPE Délégation à l'Expertise scientifique collective, à la Prospective et aux Etudes, 147 rue de l'Université, F-75338 Paris, France

2 INRA, CODIR Agriculture, 147 rue de l'Université, F-75338 Paris, France

3 IFCE, Département Recherche et Innovation, F-61310 Exmes, France

${ }^{4}$ IFCE, Direction territoriale Centre et Ile-de-France, Haras de Blois, F-41043 Blois, France

5 INRA, UMR1110 MOISA Marchés, Organisations, Institutions et Stratégies d'Acteurs, 2 place Pierre Viala,

F-34060 Montpellier, France

Courriel : Christine.Jez@paris.inra.fr

La filière équine connaît une croissance régulière depuis une trentaine d'années grâce à l'engouement des français pour l'équitation de loisir et les paris hippiques, à laquelle s'oppose, depuis peu, une forme de fragilité liée au recul des soutiens de l'Etat, au renforcement de la concurrence internationale et aux évolutions réglementaires. L'exploration des futurs possibles apporte un éclairage original pour aider les acteurs institutionnels et économiques de la filière à construire leur avenir.

\begin{abstract}
Alors que monter à cheval n'est plus une nécessité, l'équitation de loisir connaît un engouement croissant et le secteur des courses bénéficie d'un fort intérêt de la population pour les paris hippiques. Dans ce contexte dynamique, l'ouverture à la concurrence des paris en ligne, le relèvement de la TVA, la diminution des subventions de l'Etat, le développement de la concurrence internationale, mais aussi le fait que ces activités reposent majoritairement sur des micro-entreprises économiquement fragiles ou des amateurs, font peser des incertitudes sur l'avenir et sur la poursuite des tendances actuelles. Ces interrogations s'ajoutent aux nombreux enjeux auxquels la filière française est déjà confrontée tels que la pérennisation des emplois, l'intégration de nouvelles technologies comme la génomique, la production de chevaux en adéquation avec la demande, le maintien de la diversité des races et de leur présence dans certains territoires, la préservation du bien-être animal, ou encore la pérennité de la contribution du cheval au maintien de l'agriculture.
\end{abstract}

Compte tenu des nombreuses incertitudes et enjeux auxquels sont confrontés les mondes du cheval, l'Institut national de la recherche agronomique (Inra) et l'Institut français du cheval et de l'équitation (Ifce) ont décidé d'unir leurs forces et leurs réseaux pour réfléchir aux perspectives d'évolution de la filière équine à moyen terme. L'objectif est avant tout d'apporter une connaissance utile pour améliorer l'offre de services et programmer les besoins de connaissances et d'innovations de demain, mais aussi de partager cette connaissance avec les professionnels de la filière pour les aider à mieux se préparer aux enjeux d'avenir, au-delà même des éléments conjoncturels qui les mobilisent aujourd'hui. Les deux instituts ont opté pour une démarche qui accorde une attention particulière aux évolutions des futurs usages du cheval, plus propice à l'exploration de situations contrastées qu'un angle de vue centré sur la production.

Après la description de la méthode et de l'état des lieux des mondes du cheval, cet article présente les différents éléments retenus comme déterminants pour l'avenir de la filière et les scénarios prospectifs. Leur analyse transversale est ensuite exposée sous forme d'enseignements et de perspectives pour la recherche et le développement.

\section{1 / Méthodologie : l'explo- ration des futurs possibles grâce à la construction de scénarios}

La prospective est une démarche exploratoire qui a pour but d'éclairer l'action présente à la lumière de futurs possibles (Godet 1991). Grâce à la construction de scénarios vraisemblables et contrastés, elle permet d'acquérir une connaissance utile à chacun pour agir sur le futur en fonction de ses intentions et exigences. C'est donc un outil privilégié pour se préparer au changement dans les secteurs soumis à de fortes incertitudes, comme peuvent l'être les différents mondes du cheval. Plus concrètement, à travers ses enseignements et en particulier les marges de manœuvre qu'elle permet d'identifier et les débats qu'elle suscite, la prospective apporte un regard nouveau et original pour envisager l'orientation de la recherche et la définition de stratégies pour la filière. 
La maîtrise d'œuvre de l'exercice a été confiée à la Délégation à l'expertise, à la prospective et aux études de l'Inra, issue de la dynamique prospective initiée par Michel Sebillotte (Sebillotte 1993). La réflexion a été conduite par une équipe projet mixte et un groupe de travail composé d'experts provenant de différents horizons et disciplines. Ces chercheurs, représentants et acteurs économiques des différents segments de la filière se sont réunis 11 fois sur une période de 18 mois pour construire des images possibles du futur et réfléchir à leurs conséquences. L'approche collective et participative choisie crée une occasion de partager une expérience et des références communes et de construire une réflexion nécessairement interdisciplinaire. Pour renforcer la diversité des profils et des opinions, le travail de groupe a été largement complété par des auditions ciblées de 62 acteurs économiques et institutionnels de la filière et par des ateliers de réflexion dans 3 régions auxquels ont participé 42 acteurs locaux.

Le cadre de référence choisi est celui de la filière équine française, prise dans son ensemble. La filière équine est en effet constituée d'activités très diversifiées, regroupées au sein de sous-filières ou de «segments », qui comportent de nombreux acteurs n'ayant pas nécessairement d'intérêts communs ni de relations. Toutefois, il existe une certaine " porosité » entre les segments (Rossier 2004), si bien que chaque cheval a une " carrière " individualisée et peut changer de segment au cours de sa vie ; un trotteur peut par exemple débuter son parcours dans les courses, puis le poursuivre au service de l'équitation de club avant d'être orienté vers la boucherie. Compte tenu des flux d'animaux entre segments, il a donc été décidé collectivement de n'écarter aucune activité de la filière équine du cadre de référence, considérant avec un regard privilégié celles qui constituent les principaux débouchés actuels des chevaux et poneys, c'est-à-dire les segments courses, sport, loisir, tourisme, travail et viande. La « filière » est quant à elle définie comme l'ensemble des acteurs directement impliqués dans les activités équestres et hippiques, des éleveurs avec ou sans statut professionnel (agriculteur, société, simples propriétaires de juments mises à la reproduction...) aux utilisateurs finaux, qu'ils soient cavaliers, parieurs, consommateurs de viande de cheval ou simples citoyens.

L'échelle géographique sur laquelle sont étudiés les scénarios est la France, territoire sur lequel les acteurs ont la capacité de se projeter et d'élaborer des projets collectifs. Cependant, pour prendre en compte la diversité de cet espace, l'exercice s'est appuyé sur des illus- trations régionales. L'horizon temporel choisi est 2030, c'est-à-dire un pas de temps de 20 ans qui peut paraître court pour envisager des ruptures compte tenu de la lenteur des évolutions passées, mais qui témoigne de l'accélération du changement qui touche la filière, notamment du fait des évolutions politiques et réglementaires en cours, mais aussi de celle des technologies. Il est pertinent pour l'action dans la mesure où il représente l'échelle de temps sur laquelle les incertitudes actuelles sur l'évolution de certains éléments moteurs peuvent se révéler. C'est un horizon qui permet donc d'explorer des tendances en cours et des germes de changement qui pourraient devenir des sources de ruptures, tout en restant abordable pour le décideur

Le processus de construction des scénarios est fondé sur l'identification et l'exploration de tendances lourdes, de zones d'incertitudes majeures et de risques ou opportunités de ruptures. Il s'appuie sur une méthode dite « d'analyse morphologique » (de Jouvenel 1999) qui permet d'explorer les futurs possibles à partir de combinaisons d'hypothèses d'évolution des éléments déterminants pour l'avenir du système étudié. La première étape consiste à identifier les «facteurs » de toute nature qui exercent ou sont susceptibles d'exercer une influence sur la filière équine française à l'horizon 2030 et de les regrouper par grands thèmes. Ensuite, les évolutions passées et en cours sont analysées afin de définir des tendances lourdes ou émergentes et des ruptures possibles. Le groupe de travail construit alors des hypothèses d'évolution vraisemblables pour chaque facteur à l'horizon 2030. Combinées entre elles par thème, ces hypothèses constituent des « microscénarios », qui, combinés à leur tour entre eux aboutissent aux «scénarios » (Commissariat général du plan 2004).

Parmi les multiples combinaisons possibles, le choix de scénarios exploratoires contrastés s'est fondé sur les critères suivants :

- pertinence pour l'action, en ce qu'ils soulignent bien les enjeux, les incertitudes, les risques et les opportunités que la réflexion stratégique doit considérer,

- cohérence, c'est-à-dire qu'ils proposent des combinaisons logiques d'hypothèses pour donner une représentation cohérente de l'ensemble du système,

- vraisemblance,

- transparence, c'est-à-dire lisibles et justifiés,

- suffisamment contrastés pour offrir une large vision des futurs possibles.

Les scénarios issus de la combinaison d'hypothèses sont ensuite transformés en véritables histoires qui peuvent, d'une part faire apparaître des visions nouvelles et originales, et d'autre part suggérer des réflexions stratégiques grâce à la projection dans le réel (Berger 1967). Ceci étant, le récit de ces histoires force parfois le trait, jusqu'à la caricature, pour mettre l'accent sur les spécificités de chaque scénario.

Afin de nuancer les scénarios retenus par rapport aux réalités locales, leur analyse a été complétée par une illustration de leurs impacts à l'échelle de trois régions de physionomies très contrastées :

- La Basse-Normandie, première région équine par le nombre de chevaux présents, région d'élevage, d'entraînement de chevaux de courses et d'équitation.

- L'Ile-de-France, première région équestre par le nombre de licenciés à la Fédération Française d'Equitation (FFE) et première région hippique par le nombre de courses organisées chaque année.

- Le Languedoc-Roussillon, région touristique présentant un fort attachement culturel au cheval et un lien fort entre le cheval et le territoire.

Des représentants professionnels, ainsi que des acteurs économiques et territoriaux, ont été invités à imaginer les impacts des scénarios à l'échelle de leur région en termes économiques, sociaux, environnementaux et sanitaires. A partir de la construction d'illustrations locales des scénarios, le groupe de travail a analysé les impacts par type d'espace pour finalement identifier les types de territoires les plus favorables à l'expression de chaque scénario. Ces compléments apportent une dimension territoriale aux scénarios globaux qui témoigne de la diversité des situations en fonction, notamment, de la localisation urbaine/périurbaine ou rurale, de l'attractivité touristique, de l'ancrage des activités équestres dans la culture locale et de la pression sur le foncier. Les rencontres régionales ont par ailleurs permis de consolider les scénarios en confirmant leur cohérence, leur vraisemblance et leur pertinence pour la réflexion.

\section{2 / Etat des lieux de la filière équine française}

\section{1 / La filière en quelques chif- fres}

Le cheptel d'équidés français était estimé à 950000 têtes fin 2010 pour plus d'1,5 million de pratiquants (Réseau REFErences 2011b), dont plus de 700000 licenciés en 2011 (FFE 2011). La filière équine dans son ensemble rassemble 
Encadré. Près d'un million de chevaux pour des usages variés sur les territoires.

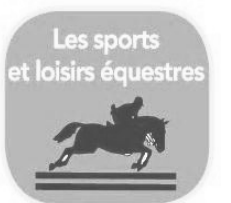

- Fédération Française d'Equitation : $3^{\text {ème }}$ fédération sportive française et $1^{\text {ère }}$ fédération féminine - 1,5 million de pratiquants dont plus de 700000 licenciés (+56\% entre 2000 et 2010$)$

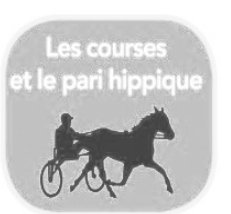

- Plus de 10 milliards d'euros de paris en 2011 (+ 60\% entre 2000 et 2010)

- 27 millions d'euros pariés / jour

- 50 courses / jour

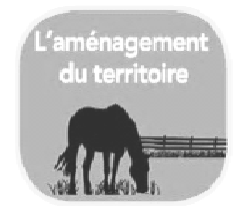

- Près d' 1 million de chevaux occupent $5 \%$ des surfaces en herbe et consomment 2 millions d'ha. de fourrages et céréales

- Entretien d'espaces, tourisme, transport, agriculture

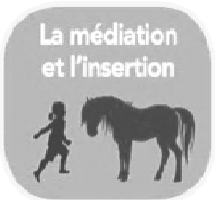

- Équithérapie : troubles physiques / psychiques

- Médiation / insertion sociale

- Rôle éducatif (enfants, citoyens...)

- Coaching en entreprise

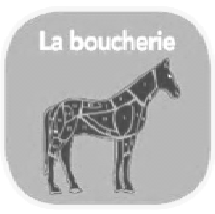

- Consommation de viande de cheval représentant en moyenne $300 \mathrm{~g} /$ personne / an, en forte baisse - Orientation bouchère en forte régression

- Cheptel trait : moins 30\% entre 2000 et 2010
53000 entreprises, essentiellement de petite taille

72000 emplois, plutôt jeunes avec un fort turn-over et une féminisation en cours

\section{2,3 Mds $€$ de chiffre d'affaires}

- dont 10 Mds $€$ de paris enregistrés par le PMU

- dont 7 Mds $€$ directement redistribués aux parieurs.

42000 propriétaires de juments reproductrices dont très peu $(15 \%)$ sont spécialisés dans l'élevage

- Ils possèdent en moyenne 2 juments

- L'élevage représente en moyenne $10 \%$ de leurs revenus

- Près de la moitié déclare ne pas générer de chiffre d'affaire

- Un cheptel en redéveloppement depuis 1995. Estimations 2008 :

- $42 \%$ de chevaux de selle

- $22 \%$ de poneys

- $19 \%$ de chevaux de courses

- $10 \%$ de chevaux de trait

- $7 \%$ d'ânes un peu plus de 53000 entreprises (Heydemann et al 2011), très diverses, dont $85 \%$ sont directement liées à la production, la valorisation ou l'utilisation du cheval (cf. encadré). Les 15\% restant sont des entreprises connexes, prestataires des entreprises précédemment citées, mais aussi de particuliers. Ces entreprises, pour la plupart de petite taille et souvent familiales, emploient environ 72000 salariés, plutôt jeunes, caractérisés par une féminisation croissante et un fort turn-over (Heydemann et al 2011). Bien que les élevages représentent $64 \%$ des entreprises, l'essentiel des emplois est concentré dans les écuries de courses et les centres équestres, les structures d'élevage ne représentant que $12 \%$ du total des emplois (Heydemann et al 2011, Réseau REFErences 2011a).

Dans son ensemble, la filière équine a généré en 2011 un chiffre d'affaires hors PMU de plus de 4 milliards d'euros, tandis que les paris hippiques, à eux seuls, représentent un peu plus de 10 milliards d'euros, dont 7 milliards sont directement redistribués aux parieurs sous forme de gains (Réseau REFErences 2012). De manière générale, le chiffre d'affaires des structures du segment courses est plus important que celui des autres segments, tandis que celui des structures d'élevage est en moyenne inférieur à celui des entreprises liées à l'utilisation du cheval. Néanmoins les écarts sont importants entre structures, notamment dans le secteur de la production où la moitié des élevages déclare ne pas générer de chiffre d'affaires (Heydemann et al 2011).

\section{2 / D'un usage militaire et agri- cole à un usage de loisir}

A la fin du XIX ${ }^{\text {ème }}$ siècle, le cheval de trait est omniprésent, à la fois dans les champs et en ville, tandis que le cheval de selle est essentiellement militaire (Digard 2004). Au XX ${ }^{\text {ème }}$ siècle, le développement des engins motorisés supplante totalement la traction animale, divisant le cheptel total d'équidés par six entre 1935 et 1970 (figure 1). Malgré la réorientation des races de trait vers la production de viande chevaline, le cheptel continue de diminuer jusqu'à la fin du XX ${ }^{\text {ème }}$ siècle (Digard 2004). Le cheptel des races de selle a quant à lui cessé de décroître grâce au développement de l'équitation de loisir, en particulier le poney depuis une vingtaine d'années, permettant même une croissance des effectifs allant jusqu'à dépasser ceux du XIX ${ }^{\text {ème }}$ siècle. Le développement de ces usages de loisir profite également, quoique dans une moindre mesure, aux races de trait, tout comme le renouveau de leur utilisation pour des missions de traction et de service public. Ces évolutions s'accompagnent d'une forte diversification des pratiques et d'une très forte féminisation et juvénilisation du public : quasi absentes au début du $\mathrm{XX}^{\text {ème }}$ siècle, les cavalières représentent $82 \%$ des licenciés en 2012 parmi lesquelles $57 \%$ ont moins de 15 ans (FFE 2012).

Les courses ont eu une évolution très différente. Bien que difficiles à dater, on sait que les courses de galop ont été importées d'outre-manche par l'aristocratie française au XVIII ${ }^{m e}$ siècle (Heydemann et al 2011) et que les premières courses de trot officielles ont eu lieu en 1836 à Cherbourg. La création du PMU, en 1930, suivie de celle du tiercé en 1954 ont permis l'essor de ces pratiques (Heydemann et al 2011).

\section{3 / Une grande variété d'usages du cheval en France}

\section{a) Les sports et loisirs équestres}

Avec plus de 700000 licenciés en 2011, dont $80 \%$ de femmes, la fédération française d'équitation est la troisième fédé- 
Figure 1. Evolution du nombre de chevaux en France (d'après Digard 2004).

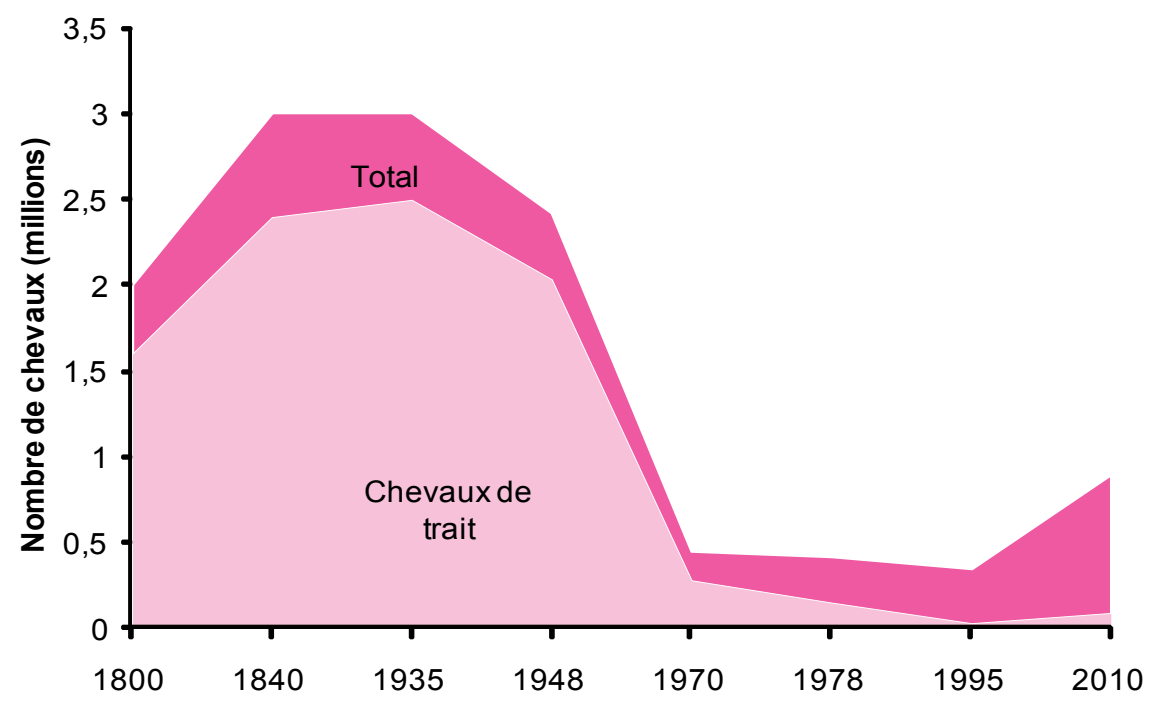

ration sportive française, mais la première fédération féminine (Ministère des sports 2011). La progression du nombre de ses licenciés est sans équivalent parmi les grandes fédérations (figure 2) et les disciplines pratiquées sont de plus en plus nombreuses. En particulier, des disciplines ludiques se développent en parallèle des disciplines olympiques. En outre, d'autres offres, telles que promenades et randonnées ou autres activités en lien avec le cheval, deviennent populaires alors que s'agrandit la population de cavaliers, propriétaires de leur monture, qui pratiquent l'équitation en dehors de toute structure. Le segment sport et loisirs représente environ 720000 équidés dont environ 435000 chevaux de sang (dont 60000 initialement produits pour la course mais réorientés vers le sport et le loisir), 200000 poneys, 20000 chevaux de trait et 60000 ânes (Heydemann et al 2011).

\section{b) Les courses et le pari hippique}

Le montant des enjeux des paris hippiques a augmenté de plus $60 \%$ entre 2000 et 2010 , en particulier grâce au développement du nombre de courses (Heydemann et al 2011) et du nombre de points de vente du PMU (Trucy 2011). En 2011, plus de 10 milliards d'euros ont été pariés sur les 18000 courses annuelles $(62 \%$ de courses de trot, $26 \%$ de courses de plat et $12 \%$ de courses d'obstacle), dont 8000 seulement sont supports de paris PMU. Quotidiennement ce sont donc 27 millions d'euros qui sont pariés sur les 20 courses supports de paris PMU. En 2011, deuxième année d'ouverture des jeux à la concurrence, seuls $1,6 \%$ du montant total des enjeux a été enregistré en ligne par les neuf opérateurs agréés hors PMU (IFCEOESC d'après Arjel et PMU). En 2008, l'Ifce a estimé le cheptel de chevaux actifs en course à près de $12 \%$ du cheptel équin français (110 000 têtes) parmi lesquels environ 30000 chevaux participent aux courses (Heydemann et al 2011).

\section{c) L'aménagement du territoire}

Les chevaux ont la capacité de s'adapter à des milieux très variés, dont des milieux difficiles ou délaissés par l'agriculture. Ils contribuent ainsi à l'entretien des espaces et à la préservation des paysages en valorisant directement environ $5 \%$ des surfaces toujours en herbe, soit 0,5 million d'hectares (Vial et al 2011). Indirectement, ils exploitent deux millions d'hectares de surfaces agricoles produisant fourrages et céréales (Réseau REFErences 2009). Selon les régions, 30 à $90 \%$ des équidés appartiennent à des particuliers indépendants et sont gérés directement par leur propriétaire sur des surfaces dont l'utilisation entre parfois en concurrence avec l'agriculture et l'urbanisation d'un point de vue foncier, mais peuvent leur être complémentaires d'un point de vue organisation des espaces. En effet, les équidés utilisent en partie des surfaces délaissées par l'agriculture mais non encore urbanisées, en particulier dans les zones périurbaines (Vial et Aubert 2011).

\section{d) La traction animale}

Dans les milieux sensibles et fragiles, et en complément d'engins motorisés, la traction animale s'avère plus adaptée, car beaucoup moins agressive que la seule utilisation d'engins motorisés (Ayache 2010). Dans un contexte de développement de l'agriculture biologique, de recherche de nouvelles pratiques culturales moins intrusives ou de développement d'une image spécifique, certaines petites structures maraîchères, viticoles ou forestières utilisent également la traction animale. En milieu urbain, le cheval contribue au tourisme urbain ou accomplit des missions de service public aussi variées que : ramassage de déchets, police, transport de personnes, entretien d'espaces verts, etc. En juillet 2012, la commission nationale des chevaux territoriaux recensait 212 communes utilisant des équidés pour des tâches variées. Ces pratiques peuvent se développer par l'achat d'un cheval en propre ou la mise en place de travail à façon.

\section{e) Médiation et insertion équine}

La médiation équine est principalement utilisée pour des jeunes enfants souffrant d'autisme et troubles apparentés, de psychoses et de troubles du comportement ou de l'attachement. Elle est également utilisée avec succès pour la rééducation de polyhandicapés (Fondation A\&P Sommer 2010). Pour les personnes en difficulté d'insertion ou de réinsertion, le cheval est un moyen d'apprendre un métier ou de réapprendre la communication avec les autres ou les règles de vie en société (Fondation $\mathrm{A} \& \mathrm{P}$ Sommer 2010). Dans les entreprises, il peut devenir un auxiliaire de coaching agissant comme un révélateur des forces et faiblesses de chacun, ainsi que du

Figure 2. Evolution du nombre de licences à la FFE depuis 1984.

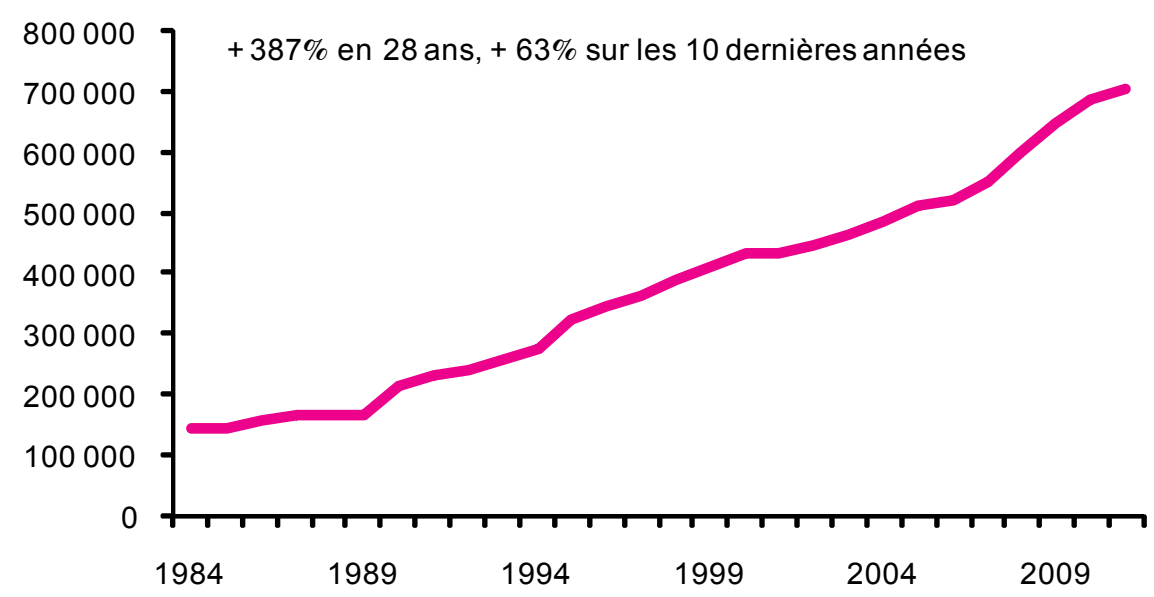


Figure 3. Les marchés de la viande chevaline en France, en milliers de Tonnes Equivalent Carcasse (TEC) (données FranceAgriMer).

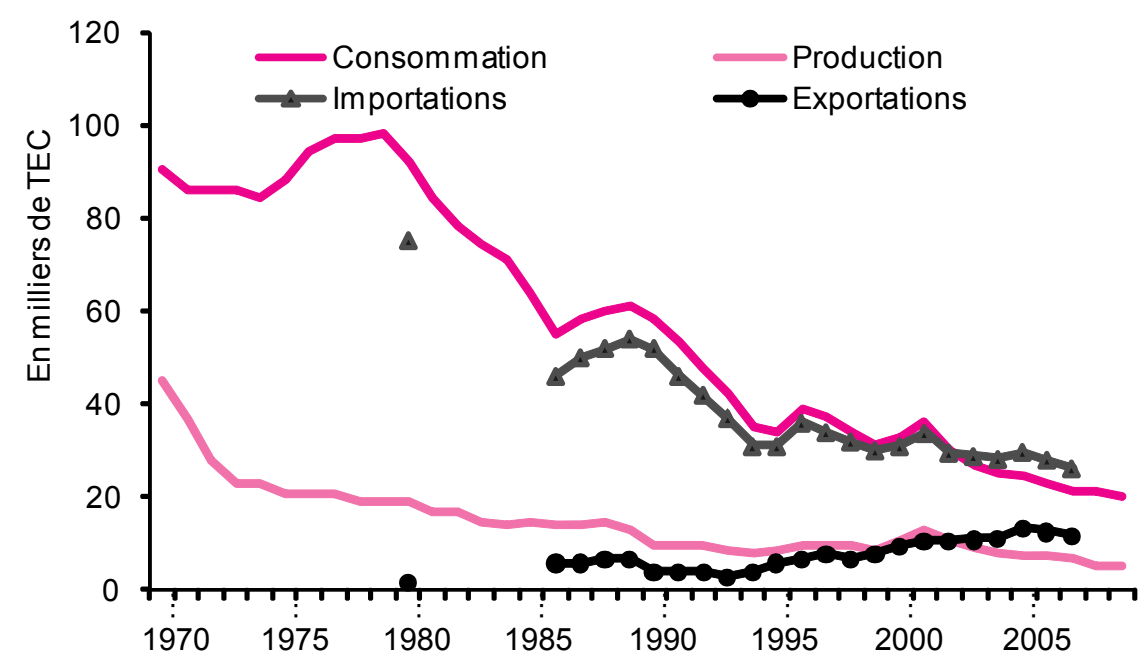

type de relation aux autres. Ces exemples laissent entrevoir un potentiel d'usages à la fois nouveaux et diversifiés.

\section{f) La viande chevaline}

La consommation de viande chevaline ne cesse de diminuer en France depuis les années 1980 (figure 3). En 2009, environ 20000 tonnes de viande chevaline ont été consommées, soit en moyenne 300 grammes par personne et par an (Heydemann et al 2011), alors que la consommation de viande bovine est de $112 \mathrm{~g}$ par personne et par jour. Moins d'un foyer sur cinq consomme de la viande chevaline, une consommation qui reste cependant occasionnelle puisqu'elle correspond annuellement en moyenne à $2,7 \mathrm{~kg}$ par foyer consommateur et par an (Pasquetti 2012). Malgré une faible demande, la production française est très inférieure à la consommation et diminue régulièrement depuis les années 1970. Deux filières coexistent: une filière d'importation de viande rouge ou d'équidés adultes principalement en provenance d'Amérique du Nord et du Sud, et une filière d'exportation de viande rosée ou de poulains, principalement vers l'Italie pour le vif et la Belgique pour la viande. En valeur comme en volume, ces échanges diminuent depuis plusieurs années mais présentaient encore un solde négatif d'environ -39,9 millions d'euros en 2010 (Réseau REFErences 2011).

\section{4 / Production et commerciali- sation}

La production de chevaux en France repose sur près de 43000 éleveurs, dont $2 / 3$ assurent la reproduction de chevaux de selle et de trait et $1 / 3$ celle de chevaux de course (Heydemann et al 2011). 80\% des éleveurs ne détiennent qu'une à deux juments (Réseau REFErences 2011) et
$85 \%$ d'entre eux, non spécialisés, déclarent tirer leurs revenus d'autres activités. Les techniques de reproduction utilisées diffèrent d'un secteur à l'autre. Ainsi, $60 \%$ des saillies de chevaux de selle sont réalisées par insémination artificielle, transportée ou congelée pour la plupart, alors que dans le secteur des courses, la monte naturelle est imposée aux Pur-Sang et l'insémination artificielle restreinte à l'utilisation de semence fraîche pour les trotteurs (Heydemann et al 2011). Dans ce secteur, la commercialisation des chevaux est structurée par les ventes aux enchères auxquelles se présentent chaque année 10 à $20 \%$ de chaque génération. Les prix de vente y varient énormément entre le type de produit et de vente, mais également au sein d'une même catégorie.

A l'opposé, le marché du cheval de selle est opaque et se caractérise par une inadéquation forte entre l'offre, principalement de chevaux de sport de haut niveau produits par des éleveurs dont l'objectif est de faire naître un champion, et la demande, principalement de chevaux de loisirs, qui ne se satisfait plus des rebuts de la filière sportive. De ce fait, les prix d'achats constatés sont en moyenne inférieurs au coût de production des animaux et le recours aux importations de chevaux s'est développé. Elles ont été multipliées par 5 en 10 ans pour les chevaux de selle et par 10 pour les poneys.

\section{5 / Organisation de la filière}

Alors que le segment courses est historiquement très structuré autour des deux sociétés mères du trot et du galop (cheval français : SECF et France Galop respectivement), elles-mêmes regroupées dans la fédération nationale des courses françaises, le segment du sportloisir-travail est encore en recherche de structuration après l'échec de la FIVAL, fédération interprofessionnelle du cheval de sport, de loisir et de travail constituée en 2005 (Heydemann et al 2011). Ainsi, la Société Hippique Française (SHF) est devenue en 2011 la société mère du cheval et du poney de sport, tandis que la Société Française des Equidés de Travail (SFET) a vu le jour en 2012. Outre ces structures collectives, de nombreuses organisations professionnelles existent dans tous les domaines, de l'élevage à la commercialisation, tant pour le sport-loisir-travail que pour les courses.

Le segment viande, quant à lui, est organisé autour de la section équine de l'interprofession du bétail et des viandes, Interbev équin, appuyée en amont par l'association France Trait, regroupant les 9 races de trait, et en aval par 19 organisations de producteurs (Heydemann et al 2011).

\section{6 / La recherche équine fran- çaise}

La recherche équine en France est assurée par de nombreux organismes publics parmi lesquels l'Inra, l'Ifce, l'ANSES, le CNRS et les écoles vétérinaires. Les laboratoires ne sont généralement pas spécialisés. Le cheval est en effet le plus souvent considéré comme un sujet d'application parmi d'autres espèces animales, ce qui contribue à la dispersion géographique et au manque de visibilité de la recherche équine française mais permet aussi des interactions très bénéfiques. Certaines équipes ont cependant acquis une notoriété internationale comme c'est le cas, d'une part de la nutrition avec la publication des tables d'alimentation et de rationnement en cinq langues (MartinRosset 1990, Martin-Rosset 2012a) et d'un précis de nutrition du cheval (MartinRosset 2012b) et d'autre part, de l'imagerie et de la kinésithérapie qui ont donné lieu à la publication d'ouvrages de référence (Denoix et Pailloux 1995, Denoix 2000).

Depuis 2011, la recherche dispose d'un nouvel outil pour travailler en partenariat avec le secteur privé à travers la fondation Hippolia, une fondation de coopération scientifique dont les objectifs sont de renforcer et de promouvoir le développement de programmes de recherche et de formation liés à la santé, au bien-être et à la performance équine.

\section{7 / Un enjeu fort : la compétiti- vité de la filière française dans un contexte d'augmentation rapide des importations}

En 2010, 10328 chevaux ont été importés, dont une petite moitié de chevaux de selle, puis $35 \%$ de chevaux de course 
et $15 \%$ de poneys (Heydemann et Dornier 2012). En dix ans, ces chiffres ont été multipliés par cinq pour les chevaux de selle et par dix pour les poneys. Certains types de chevaux sont importés car ils présentent des caractéristiques absentes dans l'offre des producteurs français. C'est le cas des chevaux de dressage (hollandais et allemands notamment) et de certains chevaux de loisir de races spécifiques (ibériques ou américains notamment). En revanche, les importations de chevaux mettent en évidence la perte de compétitivité de la production française, due en partie à ses points faibles en matière d'organisation des circuits de commercialisation et au coût élevé des intrants (saillie, échographie, soins vétérinaires...) (Heydemann et Dornier 2012, Jez et al 2012).

Ainsi, dans un marché international en plein développement et dans un contexte d'explosion de la demande de loisir, l'enjeu majeur pour la filière équine française est de parvenir à répondre à la demande de son marché intérieur. Compte tenu des difficultés économiques liées à la persistance de la situation de crise, il est également indispensable de parvenir à rentabiliser les structures existantes, en particulier celles de l'élevage. Ces principaux enjeux englobent également ceux du maintien de l'emploi et du financement de la filière, tout comme celui, très controversé, de la professionnalisation de la filière.

\section{3 / L'avenir de la filière équi- ne française : cinq grands domaines d'incertitudes}

A partir de l'état des lieux de la filière, des dynamiques d'évolution passées et des questionnements actuels sur son avenir, le groupe de travail de la prospective a recensé une quarantaine de facteurs d'évolution, susceptibles d'avoir un impact sur l'avenir de la filière équine. Ceux-ci ont été caractérisés, hiérarchisés et répartis en cinq composantes qui ont constitué la base de la construction des scénarios : 1) le contexte économique et sociétal, 2) les attentes des usagers et de la société vis-à-vis du cheval et des activités équestres et hippiques, 3) les politiques publiques et les règlementations, 4) l'offre équestre et hippique et 5) l'organisation et les stratégies des producteurs de chevaux. Ces composantes, et les éléments qui s'y rattachent, constituent les thèmes sur lesquels sont formulés des hypothèses d'avenir, à la base de la construction des scénarios (Jez et al 2012).
3.1 / Le contexte économique et sociétal : quel environnement pour la filière dans les 20 prochaines années?

L'histoire du cheval et de ses utilisations a été fortement marquée par des évolutions du contexte général, économique et sociétal, parmi lesquelles la motorisation de l'agriculture et des transports (Rossier et al 1984), l'évolution de la place du loisir dans la société, l'émancipation des femmes ou encore l'augmentation du pouvoir d'achat des classes moyennes. Dans l'avenir, d'autres paramètres pourraient avoir un rôle tout aussi prépondérant. C'est le cas, par exemple, de l'augmentation de la population mondiale qui devrait s'accompagner d'une croissance de la demande alimentaire totale entraînant une forte pression sur les terres cultivables (Paillard et al 2010) et une augmentation du prix des céréales à laquelle seront sensibles les filières animales.

A côté de ces changements globaux, considérés comme tendanciels à l'échelle mondiale dans le cadre de ce travail de prospective, les perspectives de croissance économique de la France constituent une source d'incertitude majeure. Les conséquences potentielles sur les usages du cheval, d'une crise qui toucherait plus massivement et durablement les classes moyennes de la société amènent à considérer cette hypothèse comme une possibilité à explorer. A l'opposé, un contexte de croissance économique permettant une amélioration du pouvoir d'achat des classes moyennes peut-il être suffisant pour permettre à la tendance au développement des activités de loisir de se poursuivre en s'ouvrant à de nouveaux publics? L'évolution des attentes de la société aura aussi un rôle déterminant, notamment du fait de l'extension du temps libre et de la multiplication des activités possibles pour l'occuper (Viard 2006), mais aussi de l'évolution du lien qu'entretiennent les hommes avec la nature et avec les autres hommes.

\section{2 / Une population profondé-} ment attachée au cheval, dont les attentes se recentrent sur le loisir

Le cheval alimente les rêves et désirs des hommes depuis des millénaires (Wagner 2005), et encore plus aujourd'hui alors qu'un français sur trois aimerait posséder un cheval (Haras Nationaux 2006). Ainsi, bien qu'ayant perdu son utilité en tant que force de travail, outil de guerre ou moyen de transport (Digard 2004), le cheval conserve son pouvoir attractif, même auprès des populations non cavalières. Néanmoins, sa place et son rôle dans la société n'ont cessé d'évoluer, s'adaptant aux transformations de la relation qu'entretiennent les hommes avec les animaux et la nature, et en particulier à l'engouement pour les activités de plein air, à l'émergence de préoccupations liées au bien-être des animaux ou encore à la nécessité d'un développement plus durable. Il en résulte une évolution des usages des chevaux et de la façon de s'en occuper à laquelle pourraient contribuer d'autres atouts, encore peu mis en valeur, tels que le potentiel éducatif du cheval et ses applications dans les domaines de la réinsertion de personnes en difficulté ou du handicap, par exemple.

Le public des jeux constitue un monde totalement différent et l'attirance de la société pour les paris hippiques, dans un contexte de développement des jeux d'argents sur Internet, est plus incertaine. Stimulée par une offre dynamique et créative depuis quelques années, la prise de paris sur les courses de chevaux dépend aussi du contexte économique et d'une certaine « culture de génération ».

En revanche, la consommation de viande chevaline, qui ne concerne plus qu'une très faible part de la population, offre peu de perspectives de développement dans un contexte où, par ailleurs, la consommation de viande rouge diminue légèrement en tendance depuis quelques années (FranceAgriMer 2010).

\section{3 / Une action publique en transition et des réglementations qui se renforcent autour du bien- être animal}

Les politiques publiques relatives à la filière équine sont caractérisées par des évolutions qui relèvent à la fois de politiques nationales et de l'application de politiques européennes. Il y a seulement 15 ans, l'action publique en faveur de la filière équine semblait évoluer très lentement. Le souhait d'améliorer l'efficacité des financements publics, mais aussi de respecter les réglementations européennes et de prendre en compte les évolutions des usages du cheval a peu à peu amené l'Etat à revoir ses missions et, par conséquent, sa façon d'administrer et de financer la filière (Ministères des sports, de l'agriculture et du budget 2003). Le processus engagé semble être irréversible. Il aboutit à une baisse des subventions, n'excluant pas cependant un déploiement possible des soutiens à la filière par le biais des collectivités territoriales, grâce à des compléments de financements sectoriels (fonds Eperon) (Lebrun 2010) et européens dans le cadre de la Politique Agricole Commune (PAC). Il ne signifie pas non plus la disparition d'une politique du cheval dans la mesure où l'Etat gardera 
un rôle important dans le domaine de la santé publique humaine et vétérinaire, en lien avec le cheval et ses usages. L'Etat reste également mobilisé aujourd'hui pour défendre une régulation des paris hippiques favorable au maintien de l'équilibre économique du secteur des courses, et pour accompagner la filière dans le débat qui l'oppose à l'Union Européenne sur la fiscalité des activités équestres et hippiques. Mais qu'en sera-t-il dans les 20 années à venir ?

L'action publique sera aussi de plus en plus confrontée aux évolutions des préoccupations de la société vis-à-vis du bien-être des animaux et de leurs conséquences en termes de réglementations, notamment à l'échelle de l'Europe. La prévention des risques d'accident pour les chevaux et leurs cavaliers, les conditions de détention et de transport resteront certainement des sujets majeurs alors que les évolutions des usages du cheval justifieront probablement l'élargissement des règles d'utilisation audelà des secteurs déjà très encadrés du sport et des courses. En parallèle, il semble que le débat se poursuivra sur le sujet ultime de la destination bouchère des animaux qui renvoie au transport, à l'abattage, au statut de l'animal et aux codes de conduite vis-à-vis des chevaux (Jez et al 2012).

\section{4 / L’offre équestre et hippique}

Entre la production de l'animal et son utilisation finale interviennent de multiples acteurs qui proposent des produits variés allant du cours d'équitation à la course hippique en passant par l'organisation de compétitions sportives ou de randonnées dans la nature. Parmi eux, les structures équestres jouent un rôle particulièrement important compte tenu de la popularité de l'équitation. Leur fort développement dans les dernières décennies s'est accompagné de profonds changements en termes de cavalerie, de pédagogie, d'installations mais aussi de clientèle. L'offre d' " équitation à poney » a ainsi permis de conquérir une nouvelle clientèle, très jeune et féminisée, devenue depuis majoritaire (Tourre-Malen 2009). Mais les sondages récents et les perspectives à plus long terme évoquées précédemment (cf. § 3.2) présentent de nouvelles opportunités de croissance. Ces dernières nécessitent néanmoins des adaptations en matière d'accueil et de pédagogies d'enseignement, mais aussi de coûts de prestations, dans un contexte qui pourrait devenir plus concurrentiel du fait du développement de l'offre ou plus difficile du fait du rétablissement d'une TVA à taux plein ou de la contraction de la clientèle pour des raisons économiques. Hors du cadre fédéral, l'offre équestre se développe et se diversifie notamment dans les secteurs du tourisme, de la randonnée et du spectacle équestre, grâce à un mode de vie laissant de plus en plus de place au loisir (Digard et al 2004, Vial et al 2011, Boyer 2012).

L'évolution de l'offre de courses est avant tout liée à l'évolution des paris, mais elle dépend aussi, plus indirectement, de la capacité à recruter des chevaux partants et donc de l'existence de propriétaires pour les acquérir. Si la dernière décennie a été marquée par l'accroissement modéré mais régulier $\mathrm{du}$ nombre de courses et de chevaux engagés, en relation avec une augmentation du volume des encouragements distribués, l'un et l'autre semblent malgré tout plafonner (Augereau 2012). Les hippodromes, déjà peu fréquentés par le public des courses, se diversifient ainsi dans l'organisation d'évènements non hippiques. L'équilibre global de ce segment est sensible aux évolutions du contexte économique et du volume des paris, mais aussi à la capacité du système à attribuer aux chevaux vainqueurs des allocations attractives pour leurs propriétaires.

Les offres équestres centrées sur le service à la collectivité, la médiation, la réinsertion, l'éducation, l'équithérapie ont besoin d'encouragements politiques à travers un soutien financier de la collectivité. En effet, à l'échelle des territoires, ces usages présentent des intérêts variables en termes économiques, sociaux, environnementaux et plus géneralement en termes de bien-être de la population, qui rendent difficile une approche générique.

\section{5 / La production de chevaux}

La reproduction et l'élevage des chevaux sont assurés depuis des siècles par une grande diversité d'acteurs, rarement spécialisés dans cette activité (Roche 2008). Aujourd'hui encore, pour $90 \%$ des propriétaires de juments reproductrices, élever des chevaux est un loisir avant d'être une source de revenus. Ainsi, l'activité d'élevage est principalement guidée par le plaisir de faire naître un poulain et le rêve de produire de futurs champions. Si la plupart des éleveurs destinent leurs produits à des marchés haut de gamme qui ne représentent qu'une minorité d'utilisateurs, nombreux sont ceux qui doivent vendre leurs animaux à perte dans d'autres circuits, notamment pour éviter de creuser le déficit en ayant à entretenir des chevaux qui ne feront jamais partie de l'élite (Couzy et al 2007). Ces pratiques, dans un contexte de diminution des soutiens et de développement de la concurrence d'une offre étrangère centrée sur les attentes des clients et la performan- ce technico-économique, ravivent les tensions entre les éleveurs qui essayent de vivre de leur activité et les autres.

De manière générale, l'orientation vers un élevage plus optimisé d'un point de vue économique n'est pas dans la tendance pour autant et les innovations pour améliorer la sélection, les techniques d'élevage ou l'évaluation des animaux restent diversement reçues par les acteurs de terrain. Pourtant, l'expérience passée montre que la filière s'est largement appropriée certaines innovations telles que l'échographie et l'insémination artificielle, ce qui a permis l'extension de l'aire géographique de diffusion de la génétique et l'ouverture d'un marché international (hors pursang) (Jez et al 2012).

A l'horizon de vingt ans, grâce aux progrès rapides des recherches en génomique, les connaissances auront évolué et permettront, grâce à l'analyse de leur profil génétique, une meilleure détermination des caractéristiques des animaux et donc de leur potentiel par rapport à l'exercice de certaines activités. Les éleveurs français choisiront-ils d'utiliser les nouvelles techniques pour orienter précocement les animaux vers une carrière ou une autre? Leurs concurrents étrangers augmenteront-ils leur propre compétitivité et la qualité de leurs produits en y ayant recours? Rien n'est sûr dans l'état actuel des choses. Cependant, la pression de la concurrence, la possibilité de durcissement de la crise économique ou encore l'évolution de la réglementation pourraient être des éléments déclencheurs d'un changement, d'une réflexion technique et économique vue comme une alternative à la limitation de la taille de l'élevage pour minimiser les pertes, voire d'un arrêt de l'activité.

\section{4 / Quatre scénarios explo- ratoires contrastés à l'hori- zon 2030}

A partir du questionnement sur les grands domaines d'incertitudes ayant un impact potentiel sur l'avenir de la filière, le groupe de travail a construit quatre scénarios contrastés, offrant quatre images des futurs possibles de la filière équine française à l'horizon 2030 (tableau 1). Les deux premiers scénarios « Tous à cheval» et « Le cheval des élites » sont fondés sur des évolutions opposées du contexte économique. Dans le premier, le retour du pouvoir d'achat favorise l'accès aux loisirs alors que dans le second, la persistance de la crise en limite l'accès à certaines populations. Les deux suivants, "Le cheval citoyen » et « Le cheval compagnon » 
Tableau 1. Représentation simplifiée des combinaisons d'hypothèses aboutissant aux quatre scénarios.

\begin{tabular}{|c|c|c|c|c|}
\hline $\begin{array}{l}\text { Facteurs } \\
\text { d'évolution }\end{array}$ & \multicolumn{4}{|c|}{ HYPOTHESES D'EVOLUTION A L'HORIZON 2030} \\
\hline $\begin{array}{l}\text { Contexte } \\
\text { économique } \\
\text { et sociétal }\end{array}$ & $\begin{array}{l}\text { Contexte économique } \\
\text { favorable au } \\
\text { développement du loisir }\end{array}$ & $\begin{array}{l}\text { Crise économique } \\
\text { persistante }\end{array}$ & $\begin{array}{l}\text { Priorité à la qualité de } \\
\text { vie, solidarité de } \\
\text { réseaux, quel que soit } \\
\text { le pouvoir d'achat }\end{array}$ & $\begin{array}{c}\text { Contexte économique } \\
\text { favorable au } \\
\text { développement du loisir } \\
\text { et fortes préoccupations } \\
\text { bien-être animal }\end{array}$ \\
\hline $\begin{array}{l}\text { Attentes des } \\
\text { usagers }\end{array}$ & $\begin{array}{l}\text { Désir de cheval } \\
\text { et de jeu }\end{array}$ & $\begin{array}{l}\text { Des loisirs bon marché } \\
\text { pour certains, des loisirs } \\
\text { de luxe pour d'autres }\end{array}$ & $\begin{array}{l}\text { Equilibre/harmonie, } \\
\text { grâce au lien à la } \\
\text { nature et la solidarité }\end{array}$ & $\begin{array}{l}\text { Fortes préoccupations } \\
\text { vis-à-vis du bien-être } \\
\text { animal }\end{array}$ \\
\hline $\begin{array}{l}\text { Usages } \\
\text { du cheval }\end{array}$ & $\begin{array}{l}\text { Le cheval support } \\
\text { de loisirs }\end{array}$ & $\begin{array}{l}\text { Le cheval signe } \\
\text { de distinction }\end{array}$ & $\begin{array}{l}\text { Le cheval vecteur } \\
\text { de lien social et utile } \\
\text { pour la collectivité }\end{array}$ & $\begin{array}{l}\text { La relation affective avec } \\
\text { le cheval avant tout }\end{array}$ \\
\hline $\begin{array}{l}\text { Politiques et } \\
\text { règlementations }\end{array}$ & $\begin{array}{l}\text { Un Etat régulateur } \\
\text { désengagé } \\
\text { financièrement }\end{array}$ & $\begin{array}{l}\text { Un Etat désengagé } \\
\text { de la filière équine }\end{array}$ & $\begin{array}{c}\text { Une action publique } \\
\text { décentralisée qui } \\
\text { soutient les initiatives } \\
\text { en faveur de l'utilisation } \\
\text { du cheval }\end{array}$ & $\begin{array}{l}\text { Un Etat régulateur } \\
\text { désengagé } \\
\text { financièrement }\end{array}$ \\
\hline \multirow[t]{2}{*}{$\begin{array}{l}\text { Organisation } \\
\text { et stratégies } \\
\text { des producteurs } \\
\text { de chevaux }\end{array}$} & $\begin{array}{l}\text { Forte segmentation et } \\
\text { spécialisation de la } \\
\text { production de chevaux }\end{array}$ & $\begin{array}{l}\text { La passion raisonnée, } \\
\text { professionnalisation liée } \\
\text { à la contrainte } \\
\text { économique }\end{array}$ & $\begin{array}{l}\text { La passion forcée, } \\
\text { professionnalisation } \\
\text { liée à la contrainte } \\
\text { réglementaire }\end{array}$ & $\begin{array}{l}\text { Le rêve du crack } \\
\text { et la cueillette } \\
\text { du cheval de loisir }\end{array}$ \\
\hline & $\begin{array}{l}\text { " Tous à Cheval " } \\
\text { Le cheval dans le } \\
\text { marché des loisirs } \\
\text { porté par une grande } \\
\text { diversité d'entreprises }\end{array}$ & $\begin{array}{l}\text { " Le cheval des élites » } \\
\text { Des usages restreints } \\
\text { dans une société } \\
\text { de fracture sociale } \\
\text { et de pression sur les } \\
\text { ressources naturelles }\end{array}$ & $\begin{array}{l}\text { "Le cheval citoyen " } \\
\text { Le cheval dans l'action } \\
\text { publique et collective, } \\
\text { lien entre les hommes, } \\
\text { les territoires } \\
\text { et la nature }\end{array}$ & $\begin{array}{c}\text { " Le cheval } \\
\text { compagnon " } \\
\text { De l'exploitation } \\
\text { au prendre soin, } \\
\text { la recherche du } \\
\text { bien-être de l'animal }\end{array}$ \\
\hline
\end{tabular}

sont fondés sur des évolutions de la société, marquées dans le premier par la priorité à la qualité de vie et à la solidarité, et dans le second par une évolution forte des préoccupations vis-à-vis du bien-être des animaux (Jez et al 2012).

4.1 / Scenario 1- Tous à cheval : le cheval dans le marché des loisirs porté par une grande diversité d'entreprises

Après une période de lente reprise de la croissance, le pouvoir d'achat des classes moyennes s'est amélioré. Il leur permet de concrétiser enfin leur désir de monter à cheval à tout âge, quel que soit leur niveau. L'Etat n'apportant plus de soutien direct à la filière, celle-ci est économiquement autonome pour répondre aux attentes diverses de ces usagers toujours plus nombreux. Le développement du marché des loisirs équestres a stimulé la concurrence et la différenciation de l'offre équestre pour satisfaire tous les cavaliers, du touriste occasionnel au compétiteur régulier. Par ailleurs, grâce au succès des opérations de marketing ciblé sur les jeunes parieurs, les courses sont florissantes et le PMU est leader européen de la prise de paris hippiques. Ainsi, l'élan équestre profite à l'ensemble de la filière, mais nécessite une professionnalisation généralisée et des efforts importants pour réduire les coûts tout en améliorant l'offre. Pour concurrencer les importations croissantes de chevaux étrangers, de nouveaux éleveurs se sont investis dans la production des chevaux à moindre coût, adaptés à des usages de loisir plutôt que de compétition. En réaction, les producteurs de chevaux d'élite sont incités à mieux valoriser les chevaux non retenus pour la compétition en orientant précocement chaque animal vers un type de carrière. Cette nouvelle segmentation repose sur une organisation de filière qui relie l'élevage aux attentes des usagers. Les productions de l'élevage français sont de nouveau privilégiées par les cavaliers et recherchées sur le marché international.

\section{2 / Scenario 2- Le cheval des} élites : des usages restreints dans une société de fracture sociale et de pression sur les ressources naturelles

La crise économique persistante a profondément affecté les classes moyennes dont le pouvoir d'achat s'est dégradé. En parallèle, les tensions autour de l'accès aux espaces et aux matières premières agricoles renchérissent les coûts des pratiques équestres. Les classes moyennes et populaires privilégient donc les loisirs plus abordables et l'équitation retrouve une fonction de distinction sociale qui séduit une clientèle aisée et exigeante. Les centres équestres les plus réputés et innovants se sont adaptés à ce public à la recherche d'une ambiance de club élitiste. Les difficultés économiques favorisent en revanche les jeux d'argent dont les paris hippiques. Grâce au retour d'une partie du prélèvement de l'Etat sur les enjeux, à la modernisation des outils et à l'internationalisation de la clientèle, l'économie du secteur courses se maintient. Sensible à l'augmentation de coûts structurellement déjà très élevés, les entraîneurs de chevaux de courses deviennent propriétaires, voire éleveurs, le trot restant néanmoins plus robuste que le galop. Pour sa part, le secteur viande disparaît sans trouver d'alternative à l'utilisation des chevaux de trait. En amont, l'élevage se concentre. Pour se maintenir, les éleveurs de chevaux de selle ont dû renforcer leur spécialisation et leur professionnalisation en produisant des chevaux de sport d'élite sélectionnés plus jeunes sur leur potentiel sportif ; les autres ont abandonné l'activité. Par contrecoup, une meilleure maîtrise technique et économique couplée à une orientation de carrière précoce des animaux permet à l'élevage français de s'imposer sur les marchés internationaux. 
4.3 / Scenario 3- Le cheval citoyen : le cheval dans l'action publique et collective, lien entre les hommes, les territoires et la nature

Dans un contexte de globalisation et d'accélération des rythmes de vie, la société exprime un désir croissant de nature et de rapprochement entre les personnes, auquel l'action publique territoriale répond par un soutien aux initiatives en faveur de l'utilisation du cheval au titre de l'aide à une activité économique, de la protection de l'environnement et du bien-être personnel et collectif. Les aides européennes, la décentralisation des soutiens nationaux et la restitution d'une partie du prélèvement de l'Etat sur les paris hippiques, dont il détient de nouveau le monopole, permettent la contractualisation avec des entreprises équestres privées et associatives. Si l'équitation pour tous est au cœur du dispositif, le recours au cheval pour l'entretien des espaces, l'agriculture, les services com- munaux et à la personne, l'hippothérapie, l'éducation et la réinsertion est fortement encouragé. Les hippodromes les plus fréquentés et rentables sont intégrés dans la démarche de valorisation du patrimoine équestre et hippique, les autres disparaissent. Cette multiplicité des usages permet aux chevaux de changer de carrière au cours de leur vie, ce qui limite le besoin global en animaux. Le marché français est essentiellement approvisionné par des éleveurs français qualifiés qui commercialisent des produits adaptés à la nouvelle demande au sein de réseaux de proximité valorisant signes de qualité et certificats d'aptitudes à différents usages.

\section{4 / Scenario 4- Le cheval com-} pagnon : de l'exploitation au prendre soin, la recherche du bien-être animal

En quelques décennies, la perception de la nature et des animaux a considérablement évolué au sein de la société. Le cheval est ainsi devenu un compagnon de vie, respecté pour lui-même et dont on aime avant tout prendre soin. Les propriétaires fréquentent les centres équestres pour y apprendre les soins et le comportement des chevaux, voire s'initier à des activités à pied ou à cheval pratiquées en concours. En parallèle, les revendications de la population pour le bien-être animal ont abouti à l'interdiction de la consommation de viande de cheval et au renforcement des règlementations concernant les pratiques équestres. Par conséquent, la détention et la reproduction sont règlementées tandis que les règles qui régissent les compétitions équestres et hippiques sont renforcées au prétexte d'éviter la souffrance des animaux. Le secteur courses, déjà transformé par la libéralisation totale des paris, va plus loin et organise des courses virtuelles en parallèle de celles, bien réelles, qui se disputent sur une vingtaine d'hippodromes high-tech adossés à des casinos.

Tableau 2. La filière équine à l'horizon de 20 ans selon les différents scénarios.

\begin{tabular}{|c|c|c|c|c|}
\hline & TOUS À CHEVAL & $\begin{array}{l}\text { LE CHEVAL } \\
\text { DES ÉLITES }\end{array}$ & LE CHEVAL CITOYEN & $\begin{array}{l}\text { LE CHEVAL } \\
\text { COMPAGNON }\end{array}$ \\
\hline 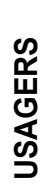 & $\begin{array}{l}\text { - Clientèle de classes } \\
\text { moyennes, tous les âges, } \\
\text { femmes et hommes. }\end{array}$ & $\begin{array}{l}\text { - Clientèle aisée, équilibre } \\
\text { hommes/femmes. }\end{array}$ & $\begin{array}{l}\text { - Bénéficiaires variés, } \\
\text { tout niveau social, } \\
\text { hommes et femmes. }\end{array}$ & $\begin{array}{l}\text { - Public varié à } \\
\text { dominante féminine sans } \\
\text { distinction sociale. }\end{array}$ \\
\hline 希 & $\begin{array}{l}\text { Cheval support } \\
\text { de loisir }\end{array}$ & 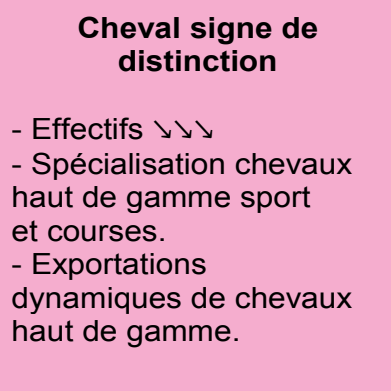 & $\begin{array}{l}\text { Cheval lien social } \\
\text { - Effectifs } \nearrow \\
\text { - Chevaux orientés, } \\
\text { éduqués et adaptés pour } \\
\text { des usages spécifiques } \\
\text { et variés. } \\
\text { - Approvisionnement } \\
\text { local. }\end{array}$ & $\begin{array}{l}\text { La relation affective } \\
\text { avant tout } \\
\text { - Effectifs \ } \\
\text { - Chevaux « coup de } \\
\text { cœur » selon } \\
\text { l'esthétique, le } \\
\text { tempérament ou la } \\
\text { régularité sportive. } \\
\text { - Approvisionnement } \\
\text { local et importations. }\end{array}$ \\
\hline$\frac{\infty}{\partial}$ & $\begin{array}{l}\text { - Salariés et travailleurs } \\
\text { indépendants } ォ \nearrow フ \\
\text { - Accueil, pédagogie, } \\
\text { animation, gestion } \\
\text { et management au cœur } \\
\text { des métiers. }\end{array}$ & $\begin{array}{l}\text { - Salariés } \searrow \searrow \text { et Moniteurs } \\
\text { indépendants } \nearrow \\
\text { - Qualifications dans } \\
\text { le domaine du luxe. }\end{array}$ & $\begin{array}{l}\text { - Salariés } \nearrow \\
\text { et bénévoles } \nearrow \nearrow \\
\text { - Compétences multiples } \\
\text { à assembler (psycho- } \\
\text { sociales et connaissance } \\
\text { du cheval, service public } \\
\text { et attelage ou } \\
\text { équitation...). }\end{array}$ & 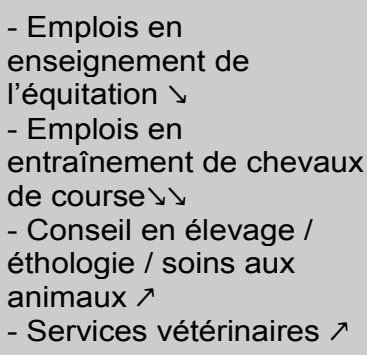 \\
\hline $\begin{array}{l}z \\
\text { 은 } \\
\frac{\delta}{0} \\
\frac{1}{d} \\
\text { 일 } \\
\text { 앵 }\end{array}$ & $\begin{array}{l}\text { - Zones touristiques ; } \\
\text { lieux de villégiature } \\
\text { des urbains. } \\
\text { - Régions dynamiques. } \\
\text { - Régions d'élevage } \\
\text { cavalières. } \\
\text { - Développement limité } \\
\text { par la pression foncière } \\
\text { et les temps de transport } \\
\text { à proximité des grandes } \\
\text { métropoles. }\end{array}$ & $\begin{array}{l}\text { - Zones touristiques } \\
\text { fréquentées par les élites. } \\
\text { - Zones spécialisées dans } \\
\text { l'élevage de chevaux de } \\
\text { haut niveau. } \\
\text { - Migration de l'élevage } \\
\text { et de l'entraînement vers } \\
\text { les zones à moindre } \\
\text { pression foncière. }\end{array}$ & $\begin{array}{l}\text { - Développement plus ou } \\
\text { moins fort des activités } \\
\text { en fonction des projets, } \\
\text { spécificités et cultures } \\
\text { locales des territoires. }\end{array}$ & $\begin{array}{l}\text { - Toutes zones rurales ou } \\
\text { périurbaines si espaces } \\
\text { herbagers. } \\
\text { - Sanctuaires pour } \\
\text { chevaux menacés de } \\
\text { disparition ou retraités en } \\
\text { fin de vie. }\end{array}$ \\
\hline
\end{tabular}


L'élevage est avant tout un loisir de propriétaires particuliers. Néanmoins, une partie des éleveurs, passionnés autrefois par la performance, produit des chevaux à la fois capables de présenter de longues carrières sportives et des caractéristiques esthétiques spécifiques. Un marché de chevaux inactifs s'est développé sur Internet et répond au besoin grandissant de chevaux compagnons. La population équine vieillit et se renouvelle très lentement.

Les quatre scénarios présentés tracent des physionomies de la filière très contrastées à horizon de 20 ans. Ils conduisent à des effectifs d'équidés différents : peut-être deux fois plus qu'aujourd'hui dans le « Tous à Cheval » ou deux fois moins dans le « cheval des élites ». Les entreprises, les types d'emplois différeraient également beaucoup (cf. ceux du " Cheval citoyen »), de même que la localisation des chevaux dans les régions et zones rurales françaises (tableau 2).

\section{5 / Principaux enseigne- ments de la réflexion pro- spective}

\section{1 / Les questions transversales soulevées par les scénarios}

Parmi les préoccupations communes aux différents scénarios figure en premier lieu la relation de l'homme au cheval, un élément central à prendre en compte et à mieux connaître. Ce lien, présent sous des formes différentes dans tous les scénarios, est révélateur de l'évolution de la place du cheval dans la société. Mieux connaître les besoins et attentes des différents publics en relation avec les chevaux est une nécessité commune.

L'efficacité économique de la filière est aussi largement questionnée. Tous les scénarios sont confrontés à des préoccupations fortes en matière de coûts de production des chevaux et des services équestres, que ce soit pour diversifier l'offre, améliorer la qualité, attirer de nouveaux usagers, être plus compétitif au niveau international, survivre hors du luxe en temps de crise, faire face à des coûts d'aliments et d'énergie croissants, favoriser certains usages - coûteux - du cheval, entretenir des animaux vieillissants et maintenir leur santé et leur bien-être, gérer l'élimination des cadavres, etc. ou encore faire face à une hausse de la TVA. Sans changement, le développement des activités risque d'être très dépendant de variations du contexte économique et d'offres étrangères plus compétitives.
Tous les scénarios sont confrontés à la montée des enjeux environnementaux et de pression sur les ressources. La filière a des atouts, liés à l'avantage comparé des équidés sur les autres herbivores en matière d'émissions de gaz à effet de serre, d'utilisation des surfaces en herbe, de contribution à la qualité des paysages. En revanche, les risques d'érosion de la diversité des races équines sont réels : la menace actuelle sur les races de chevaux de trait pourrait s'étendre du fait du développement d'une approche strictement commerciale favorisant la perte de cheptels en race pure. La concurrence entre activités consommatrices d'espace prendra aussi plus d'acuité dans un contexte de pression croissante sur les ressources naturelles et l'utilisation du sol.

La santé, le bien-être animal, la fin de vie des animaux sont aussi des questions récurrentes. La tendance lourde commune est une demande accrue de médicalisation, de surveillance sanitaire, de bien-être animal. Les scénarios ne sont pas discriminants par rapport aux risques de développement de nouvelles maladies, qui peuvent émerger et se diffuser sur le territoire quels que soient les effectifs de chevaux. Le vieillissement des chevaux, la gestion de la fin de vie sont des problèmes majeurs qui nécessiteront une approche spécifique sur la bientraitance et des solutions alternatives à l'abattage ou l'équarrissage.

\section{2 / Les perspectives pour la recherche et le développement}

Il convient d'abord de rappeler que, depuis les années 1970, beaucoup de connaissances, de méthodes et d'outils ont été acquis par la recherche publique, mais que le transfert de ces connaissances aux utilisateurs demeure très insuffisant malgré la création, comme chez les autres espèces, d'un réseau de techniciens de développement spécialisés équins. Dans cette filière, où les éleveurs sont principalement constitués de particuliers pour qui la reproduction est un loisir, la vulgarisation et le conseil sont confrontés à la dispersion du cheptel reproducteur et aux difficultés à atteindre les bénéficiaires. L'arrêt de l'étalonnage public ne va pas non plus dans le sens d'une amélioration des contacts entre éleveurs et circuits de vulgarisation des techniques. D'autres voies sont donc à inventer ou à renforcer. Ces constats et l'ensemble de la réflexion issue de la prospective débouchent sur les besoins généraux de recherche suivants, qui appellent quelques priorités qui seront abordées en conclusion. a) Mieux connaître le comportement animal et relation homme-animal

Une première direction de recherche transversale dont la prospective appelle le développement est celle du comportement du cheval et de la relation hommeanimal. La caractérisation du tempérament est une thématique de recherche à développer, commune aux quatre scénarios : qu'il s'agisse de sélectionner des chevaux calmes adaptés aux cavaliers de petit niveau (club, randonnée) qui représenteraient la majorité des utilisateurs du « Tous à Cheval », ou d'éviter d'élever des chevaux qui ne trouveront pas de débouchés en raison d'un tempérament inadapté dans "Le cheval des élites », ou encore d'écarter les peureux et les agressifs qui, dans les usages du « Cheval citoyen », seraient en contact avec des populations qui ne connaissent pas ou peu cet animal, ou enfin d'éviter les accidents domestiques chez les nombreux novices possédant un «Cheval compagnon ».

La recherche pourrait/devrait aussi se donner comme objectif de contribuer à la mise au point d'indicateurs de bienêtre animal et de déterminer les conditions qui le favorisent. Les deux scénarios du « Cheval citoyen » et du « Cheval compagnon » nécessitent plus généralement d'approfondir la connaissance de la relation homme-animal et du comportement naturel du cheval, afin de répondre à ce besoin de nature et d'harmonie entre les hommes et les animaux. Une meilleure compréhension de la relation de l'animal à l'homme pourrait également permettre d'améliorer les techniques d'éducation des chevaux dans l'objectif d'en améliorer l'efficacité.

\section{b) Mieux connaître les acteurs et} opérateurs de la filière

Il apparaît également comme une nécessité impérieuse d'acquérir une meilleure connaissance des acteurs et des opérateurs de la filière équestre. Les questions soulevées par la prospective soulignent ce besoin fort d'une meilleure connaissance technique, économique et sociale du fonctionnement des activités équestres et de leurs acteurs et utilisateurs. Des recherches en amont en sociologie et en marketing stratégique sont nécessaires pour comprendre les représentations, les usages, les attentes et les relations des diverses clientèles à l'égard des chevaux. En économie, en gestion et en sociologie, des recherches de portée plus générale associées à des travaux très appliqués de recherchedéveloppement, tels qu'ils sont engagés à l'Institut de l'élevage sur les systèmes d'élevage avec la production de référentiels, sont nécessaires pour alimenter les réflexions sur l'organisation du travail dans les structures équestres, ainsi que 
sur les questions de coûts de production et d'entretien des chevaux. La recherche et la R\&D devraient contribuer à ce que les opérateurs puissent introduire les innovations, les changements de pratiques qui permettraient d'améliorer la compétitivité des entreprises et/ou une meilleure adaptation de celles-ci à la demande.

c) Produire des chevaux qui correspondent aux usages

Plus spécifiquement, la prise en compte des attentes des utilisateurs autour du cheval de loisir ou de ses usages territoriaux ou thérapeutiques, tels qu'ils s'expriment dans les scénarios du «Tous à cheval » ou du «Cheval citoyen ", supposent une inflexion et une adaptation des recherches en matière d'amélioration génétique des équidés. L'intérêt des outils modernes de la génétique est évident pour améliorer la performance sportive de l'animal en compétition, mais il doit se développer aussi pour servir d'autres usages du cheval selon des critères d'évaluation différents. Dans chaque scénario, la sélection de types génétiques adaptés et souvent très spécialisés est un levier de la modernisation et de la plus ou moins grande intensification de la production. Elle nécessite une définition plus claire des objectifs de sélection, des innovations dans les outils et les méthodes, une plus solide organisation collective des acteurs et une acceptation sociale moins frileuse de ces technologies, associée à d'autres outils plus classiques.

L'espèce équine ne peut demeurer à l'écart de ces innovations, incluant la génomique. Au contraire, cette filière peut retrouver un leadership européen, voire mondial, dans ce domaine en favorisant une évaluation plus précise de la valeur génétique des animaux et une caractérisation précoce des potentialités des poulains permettant une optimisation de leur conduite, y compris dans le secteur des courses. L'importance pour les éleveurs de disposer d'une information partagée, fiable et neutre sur la qualité des reproducteurs devient primordiale pour le maintien d'un progrès génétique. La génomique permet en outre la validation de quelques biomarqueurs en lien avec la réponse à l'entraînement ou certaines pathologies, voire pour la lutte contre le dopage. Il faut aussi souligner l'importance du phénotypage précoce, à grande échelle, qui nécessitera de définir des critères, de développer des outils, de valider les orientations prises.

d) Optimiser la conduite d'élevage et l'impact environnemental des équidés

Dans une perspective de développement durable, l'optimisation des coûts et du bien-être des chevaux appelle un autre axe de recherche, centré sur la thématique de la conduite d'élevage. Il consisterait, entre autres, à évaluer les capacités du cheval à utiliser au maximum les aliments les moins coûteux, l'herbe pâturée et les fourrages conservés, pour satisfaire ses besoins nutritionnels au cours des cycles successifs d'élevage et d'utilisation. Corrélativement, cela devrait déboucher sur des programmes visant à l'évaluation de l'impact environnemental des chevaux, selon leurs différents types d'utilisation, de conduite et de localisation en s'inspirant pour cela de caractéristiques qui sont à l'œuvre dans les différents scénarios pour ces critères. L'ensemble de ces résultats serait transférable aux entreprises équestres et agricoles ainsi qu'aux particuliers sur la base de référentiels technico-économiques. Pour cela, il faut d'une part, que les connaissances en matière de conduite d'élevage des animaux et de gestion des surfaces fourragères soient mieux transférées et d'autre part, que la gestion génétique des races équines adaptées aux différents milieux soit mise en place, notamment pour les populations à faible effectif comme les races rustiques.

Plus largement, les évolutions de la PAC favorisant une agriculture plus respectueuse de l'environnement et une part plus grande des aides au développement rural peuvent générer des travaux de recherche pour caractériser les services écosystémiques apportés par la filière cheval, en particulier autour de l'entretien et la préservation de surfaces en herbe par les chevaux. Les équidés possèdent de nombreux atouts, complémentaires de ceux des ruminants, pour préserver ou augmenter la biodiversité des prairies. Les marges de progrès dans l'exploitation des fourrages, notamment l'herbe, par les chevaux sont importantes. Des stratégies innovantes de conduite des animaux et d'utilisation de la prairie restent à élaborer. L'utilisation par les chevaux de certaines de ces surfaces qui risquent d'être de plus en plus délaissées par l'exploitation des ruminants pourrait faire l'objet de travaux expérimentaux dans le cadre de systèmes d'élevage moins intensifs, facilitant le regroupement de troupeaux et donc des modes de conduite moins onéreux, freinant des risques de délocalisation hors de France. C'est peut-être l'atout du scénario « Tous à Cheval» dans une logique de réduction des coûts et d'une production adaptée à l'aval en réponse à un marché de masse.

\section{e) Mieux gérer la santé des équidés}

Les quatre scénarios appellent enfin un développement des recherches en matière de santé animale. Santé, bien- être et performances sont liés et ceci pour toutes les utilisations du cheval. Toutefois, les besoins de recherche en santé animale se différencient selon les scénarios : pour « Tous à Cheval», la sécurité sanitaire prime, parallèlement bien évidemment à celle de la sécurité des utilisateurs (recherche sur les matériels - casques, gilets de protection, lisse de sécurité - développement de pédagogie permettant d'assurer la sécurité du cavalier...). Les études d'épidémiosurveillance et la mise en ouvre de protocoles de prévention sont indispensables, notamment au vu de l'accroissement des échanges européens et internationaux et du réchauffement climatique augmentant le risque de maladies transmises par les insectes. Pour « Le cheval des élites », l'accent sera davantage mis sur tout ce qui améliore la performance (suivi médico-sportif de l'athlète, préservation de la santé), quitte à avoir une utilisation plus abondante de pharmacologie. Pour le « Cheval compagnon », mais aussi plus transversalement pour les autres scénarios, le vieillissement des chevaux, l'accès à la " retraite " et la gestion de la fin de vie sont et seront des problèmes majeurs. A vouloir maintenir un cheval en vie à tout prix, on peut l'amener à de très grandes souffrances.

\section{Conclusion}

Bien que les différents segments de la filière équine aient des logiques de fonctionnement autonomes, et que les scénarios aboutissent à des futurs possibles très contrastés, des questions communes ont pu être mises en évidence, accordant un poids important à la place du cheval dans la société, à la nécessité de maîtrise des coûts à différents niveaux, à l'impact environnemental du cheval, aux préoccupations liées au bien-être des animaux et au risque d'érosion de la biodiversité équine. Ces éléments ne signifient cependant pas que la filière représente une unité derrière ses cloisonnements et ses interactions, mais que sur certains thèmes, les réflexions peuvent être partagées.

Par rapport au panorama actuel des recherches, la prospective conforte en partie les orientations de la recherche publique déjà existantes, mais insuffisamment transférées ou appropriées. Elle souligne aussi, d'une part, la nécessité d'infléchir la hiérarchie actuelle des recherches : la montée en puissance des travaux dans les domaines économique, social, environnemental, ainsi que sur le comportement animal est indispensable. D'autre part, elle souligne la nécessité de repenser le transfert des connaissances et des innovations pour que ces dernières puissent être plus largement 
adoptées. Dans un contexte international où ces avancées sont déjà prises en compte par d'autres éleveurs soucieux d'une meilleure rentabilité économique, les éleveurs français pourront difficilement rester à l'écart de celles permises par la génomique. Ces innovations seront utiles aussi bien pour l'amélioration de la performance sportive, que pour avoir accès à d'autres caractères, comme le tempérament ou certaines pathologies, particulièrement intéressants pour l'élevage et la relation homme-cheval.

Les perspectives pour la recherche présentées constituent des marges de manœuvre pour faire face aux évolutions à venir. Elles seront discutées au cours de l'année 2013 par le Conseil scientifique de l'Ifce et le Groupe filière équine de l'Inra, chargés par les directions des deux instituts d'établir un document d'orientation de la recherche équine pour les prochaines années. En parallèle se poursuivra la diffusion des résultats de l'exercice auprès d'acteurs économiques et institutionnels afin d'initier et de stimuler la réflexion individuelle et collective, mais aussi de permettre aux chercheurs d'interagir avec les utilisateurs et les bénéficiaires potentiels de leurs travaux dans le cadre d'échanges autour de la prospective.

\section{Remerciements}

Les auteurs remercient le groupe de travail ainsi que l'ensemble des personnes ayant accepté de contribuer à ce travail en participant à un atelier ou en acceptant d'être auditionné. Ils remercient les relecteurs.

\section{Références}

Augereau D., 2012. L'institution des courses : la réforme incontournable d'un modèle d'avenir. Rapport remis à François Fillon, Premier ministre, 96p.

Ayache S., 2010. Energie animale et gestion des espaces naturels. ONF Agence Ain-LoireRhône, Les cahiers techniques, Bourg-enBresse, France, 20p.

Berger G., 1967. Étapes de la prospective. PUF, Paris, France, 343p.

Boyer C., 2012. Les infrastructures du tourisme équestre, séminaire équi-meeting tourisme. In : Cheval, tourisme et loisirs, transformations, permanences et ruptures. Séminaire international 9-11 mai 2012, Ecole Nationale d'Equitation, Saumur, France, 167-168.

Commissariat général du plan, 2004. La méthode des scénarios, outil d'une démarche prospective. Paris, France, 16p.

Couzy C., Capitain M., Palazon R., Coquan M., 2007. Peut-on encore parler d'éleveur, de cheval ou d'équitation de sport ou de loisir? $33^{\text {eme }}$ Journ. Rech. Equine, 8 mars 2007, Les Haras Nationaux, Paris, France, 119-132.

De Jouvenel H., 1999. La démarche prospective, un bref guide méthodologique. Futuribles, 247, 24p.

Denoix J.M., 2000. The equine distal limb: an atlas of clinical anatomy and comparative imaging. Manson Publishing Ltd, London, UK, 390 p.

Denoix J.M., Pailloux J.P., 1995. Physical therapy and massage for the horse. Manson Publishing Ltd, London, UK, 200p.

Digard J.P., 2004. Une histoire du cheval. Art, techniques, société. Actes Sud, Arles, France, 232p.

Digard J.P., Ould-Ferhat L., Tourre-Malen C., Caporal A., Vialles N., 2004. Cultures équestres en crise : professionnels et usagers du cheval face au changement. Les Haras Nationaux, Compte rendu final d'activité 2001-2003. 41p.

Fédération française d'équitation, 2011. Statistiques licences.

Fédération française d'équitation, 2012. Statistiques licences.

Fondation A\&P Sommer, 2010. Un cheval pour vivre. Dossier pour le colloque «Un cheval pour vivre» au Muséum National d'Histoire Naturelle, 10 Juin 2010, Paris, France, 13p.

FranceAgriMer, 2010. La consommation française de viandes. Evolutions depuis 40 ans et dernières tendances. Les synthèses de FranceAgriMer, 8p.

Godet M., 1991. De l'anticipation à l'actionManuel de prospective et de stratégie. Dunod, Paris, France, 390p.

Haras Nationaux, 2006. Annuaire ECUS 2006. Les Haras Nationaux, Le Pin au Haras, 63p.

Heydemann P., Dornier X., 2012. Le marché du cheval de sport-loisir en 2010, synthèse des résultats de l'enquête menée en 2011 auprès de 4000 acheteurs. IFCE, Observatoire économique et social du cheval, $14 \mathrm{p}$.

Heydemann P., Boyer S., Couzy C., Dornier X., Madeline L., Morhain B., Ragot N., 2011. Panorama économique de la filière équine. Les Haras Nationaux, Le Pin-au-Haras, France, $241 \mathrm{p}$.

Jez C., Coudurier B., Cressent M., Méa F. Perrier-Cornet P., Rossier E., 2012. La filière équine française à l'horizon 2030. Rapport du groupe de travail de la prospective équine. Inra-Ifce, Paris, France, 98p.

Lebrun J., 2010. Les enjeux et les perspectives de la filière équine en France. Paris, Conseil économique, social et environnemental, 29p.

Martin-Rosset W., 1990. L'alimentation des chevaux. Inra Editions, Versailles, France, 232p.

Martin-Rosset W., 2012a. Alimentation des chevaux. Table des apports alimentaires Inra 2011, Editions Quae-Haras Nationaux, Versailles, France, 264p.

Martin-Rosset W., 2012b. Nutrition et alimentation des chevaux. Editions Quae, Versailles, France, 624p.

Ministère des Sports, Ministère de 1'Agriculture, Ministère du budget, 2003. Une nouvelle politique pour le cheval. Le Pin-auHaras, France, 28p.

Ministère des Sports, 2011. Répartition des licences masculines et féminines par fédération française agréée (hors ATP). Statistiques, données détaillées, page consultée le 20/04/2012, http://www.sports.gouv.fr/index/communica- tion/statistiques/donnees-detaillees/donneesdetaillees-2011

Paillard S., Treyer S., Dorin B., 2010. Agrimonde, scénarios et défis pour nourrir le monde. Editions Quae, Versailles, France, 296p.

Pasquetti C., 2012. Aperçu du marché de la viande chevaline. Interbev, $5 \mathrm{p}$. Pages consultées le 20/01/2013, http://www.interbev.fr/ fileadmin/docs/marche-2011-viande-chevaline.pdf

Réseau REFErences, 2009. Caractéristiques socio-économiques des entreprises de la filière équine: Les Observatoires Economiques Régionaux, 16p.

Réseau REFErences 2011a. Annuaire écus 2011, Tableau économique, statistique et graphique du cheval en France, données 2010/ 2011, Institut Français du cheval et de l'équitation, 63p.

Réseau REFErences, 2011b. Filière équine, chiffres clés 2011, Institut français du cheval et de l'équitation, $6 \mathrm{p}$.

Réseau REFErences, 2012. Filière équine, chiffres clés 2012, Institut français du cheval et de l'équitation, 6p.

Roche D., 2008. La culture équestre de l'Occident XVI-XIX siècle. L'ombre du cheval. Tome premier-Le cheval moteur. Editions Fayard, Paris, France, 479p.

Rossier E., 2004. "La filière hippique en France : quelques repères et chiffres clés." Bull. Acad. Vet., 157, 15-22.

Rossier E., Coleou J., Blanc H., 1984. Les effectifs de chevaux en France et dans le monde. In : Le cheval : Reproduction, sélection, alimentation, exploitation », $13^{\text {eme }}$ Journée du Grenier de Theix, INRA, Paris, France, 11-24.

Sebillotte M., 1993. Avenir de l'agriculture et futur de l'INRA, INRA, Paris, France, 139p.

Tourre-Malen C., 2009. Évolution des activités équestres et changement social en France à partir des années 1960. Mouvement Social 4/2009, 229, 41-59.

Trucy F., 2011. Evaluation de la loi n²010$476 \mathrm{du} 12$ mai 2010 relative à l'ouverture à la concurrence et à la régulation du secteur des jeux d'argent et de hasard en ligne. Paris, Sénat, $315 \mathrm{p}$.

Vial C., Aubert M., 2011. Quand les équidés de particuliers indépendants gagnent du ter- 
rain... Focus sur les déterminants d'une nouvelle utilisation des territoires. $5^{\text {emes }}$ Journ. Rech. Sci. Soc., INRA - SFER - CIRAD, AgroSup Dijon, France, 26p.

Vial C., Perrier-Cornet P., Soulard C., 2011. Le développement des équidés de loisir en
France : quels impacts sur les espaces ruraux et périurbains? Fourrages, 207, 165-172.

Viard J., 2006. Eloge de la mobilité. Essai sur le capital temps libre et la valeur travail. Edition de l'Aube, Paris, France, 205p.
Wagner M.A., 2005. "Le cheval dans les croyances germaniques : paganisme, christianisme et traditions. Champion-Nouvelle bibliothèque du Moyen Age $\mathrm{N}^{\circ} 73$, Paris, France, 974p.

\title{
Résumé
}

Pour programmer les besoins de connaissances et d'innovations de demain, mais aussi aider les professionnels à anticiper les évolutions à venir, l'Institut national de la recherche agronomique et l'Institut français du cheval et de l'équitation se sont investis dans une exploration de l'avenir de la filière équine française grâce à la construction de scénarios contrastés à l'horizon 2030. L'exploration des différents futurs possibles apporte un regard nouveau et original dans un contexte où s'opposent une dynamique de développement liée à l'engouement des français pour les activités équestres et hippiques et une forme de fragilité du fait du recul des soutiens de l'Etat, du renforcement de la concurrence internationale, des évolutions de la société vis-à-vis du bien-être des animaux et de l'harmonisation des réglementations à l'échelle européenne. L'analyse des évolutions passées, des tendances en cours et des ruptures possibles a abouti à la construction de quatre scénarios caractérisés par des usages, des effectifs d'équidés et des emplois très différents. Ceux-ci soulèvent cependant des préoccupations communes en termes de $i$ ) relation de l'homme au cheval, ii) d'efficacité économique, iii) d'enjeux environnementaux, $i v$ ) de préservation des races et de pression sur les surfaces, et $v$ ) de santé, de bien-être animal et de gestion de la fin de vie des animaux. Ces questions appellent un développement des recherches dans les domaines du comportement animal, des sciences économiques et sociales, de la conduite d'élevage et de l'amélioration génétique. Elles soulignent également la nécessité de repenser et d'améliorer le transfert des connaissances et des innovations.

\begin{abstract}
Prospects for the French equine industry in 2030. Four contrasting scenarios to inform decision-making

To plan for the knowledge and innovation needs of tomorrow, but also to help stakeholders anticipate future developments, the French National Institute for Agricultural Research (INRA) and the French Institute for Horses and Horse Riding (IFCE) have joined forces to conduct a scenario building exercise for the French equine industry in 2030. Exploring different futures sheds new light on a context which opposes increasing popular interest in equestrian activities and horse racing, and difficulties due to the decline of State support, the increase in international competition, societal changes regarding animal welfare and the harmonization of regu-lations at the European level. The analysis of past and current trends, and potential shifts thereof has led to four scenarios characterized by different horse usages, different horse populations, and jobs. However, they raise shared concerns in terms of $i$ ) the relationship between man and the horse, ii) economic efficiency, iii) environmental issues, iv) preservation of breeds and pressure on surfaces, and v) health, animal welfare and looking after animals up to and beyond death. These questions call for research development in the fields of animal behaviour, economic and social sciences, breeding and genetic improvement. They also emphasize the need to improve knowledge and innovation transmission.
\end{abstract}

JEZ C., COUDURIER B., CRESSENT M., MÉA F., PERRIER-CORNET P., 2013. Prospective de la filière équine française à l'horizon 2030. Quatre scénarios contrastés pour éclairer la décision. INRA Prod. Anim., 26, 1, 51-64. 